\title{
Wie plausibel ist die Kontinuumshypothese?
}

\author{
Gerald Kuba \\ Gerald Kuba schloss 1985 sein Mathematik-Studium an der Universität Wien ab, um \\ danach als wissenschaftlicher Mitarbeiter an der wirtschaftstheoretischen Fakultät der \\ Universität Bielefeld tätig zu sein. Er promovierte 1991 an der Universität Wien und ist \\ seit 2001 Dozent für Mathematik ebendort, sowie außerordentlicher Professor an der \\ Universität für Bodenkultur Wien, wo er seit 1994 beschäftigt ist. Sein Hauptinteresse \\ gilt der analytischen Zahlentheorie, speziell der Gitterpunktlehre, sowie elementarma- \\ thematischen Themen.
}

\section{Einleitung}

Eine Menge $\mathcal{A}$ heißt abzählbar, wenn $\mathcal{A}$ gleichmächtig mit irgendeiner Teilmenge von $\mathbb{N}$ ist. Sind zwei Mengen $X$ und $Y$ gleichmächtig, läßt sich also $X$ bijektiv auf $Y$ abbilden, so schreiben wir $|X|=|Y|$. Unendliche Mengen, die abzählbar sind, sind stets gleichmächtig mit $\mathbb{N}$ und heißen abzählbar unendlich. Ist eine Menge $A$ mächtiger als eine Menge $B$, läßt sich also $B$ injektiv in $A$, nicht aber bijektiv auf $A$ abbilden, so schreiben wir $|A|>|B|$ bzw. $|B|<|A|$. Für zwei beliebige Mengen $X$ und $Y$ gilt stets entweder $|X|<|Y|$ oder $|X|=|Y| \operatorname{oder}|X|>|Y|$. Eine Menge $M$, für die $|M|>|\mathbb{N}|$ gilt, heißt überabzählbar.

Das erstmalig von Cantor im Jahr 1878 formulierte Kontinuumsproblem stellt die Frage, ob es zwischen der Kardinalität der Menge $\mathbb{N}$ der natürlichen Zahlen und der Kardinalität des Zahlenkontinuums $\mathbb{R}$ weitere Kardinalzahlen gibt. Die negative Antwort auf diese Frage, also die Behauptung, dass jede überabzählbare Teilmenge von $\mathbb{R}$ von derselben Kardinalität wie $\mathbb{R}$ ist, nennt man die Kontinuumshypothese. Nach den berühmten Sätzen von Gödel und Cohen ist diese Hypothese im Rahmen einer axiomatischen Mengenlehre weder beweisbar noch widerlegbar. Allerdings widerstrebt es vielen Mathematikern, in diesem Unabhängigkeitsresultat eine endgültige Lösung des Kontinuumsproblems zu sehen. Vielmehr weisen die Ergebnisse von Gödel und Cohen darauf hin, dass die axiomatische Mengenlehre den intuitiven Mengenbegriff (noch) nicht zufriedenstellend erfasst. In diesem Übersichtsartikel präsentiert der Autor allgemein verständlich (ohne Verwendung von Ordinal- bzw. Kardinalzahlen) einige der interessantesten Hypothesen der Analysis und stellt damit die Plausibilität der Kontinuumshypothese zur Diskussion. 
Das wichtigste Beispiel einer überabzählbaren Menge ist das Zahlenkontinuum $\mathbb{R}$. Die spezielle Kontinuumshypothese - im folgenden stets mit $(\mathrm{KH})$ abkürzend bezeichnet besagt:

(KH) Wenn $\mathbb{N} \subset X \subset \mathbb{R}$, dann gilt stets entweder $|X|=|\mathbb{N}|$ oder $|X|=|\mathbb{R}|$.

Ergänzend führen wir noch zwei alternative Formulierungen von $(\mathrm{KH})$ an:

(KHa) Für alle Mengen $M$ ist die Kettenungleichung $|\mathbb{N}|<|M|<|\mathbb{R}|$ falsch.

(KHb) Ist U eine überabzählbare Teilmenge von $\mathbb{R}$, dann gilt $|U|=|\mathbb{R}|$.

\section{Der Potenzmengensprung}

Das Kontinuum $\mathbb{R}$ ist bekanntlich gleichmächtig mit der Potenzmenge von $\mathbb{N}$. Allgemein ist die Potenzmenge $\mathcal{P}(M)$ einer Menge mächtiger als die Menge $M$ selbst: $|\mathcal{P}(M)|>|M|$ (Satz von CANTOR). Die sog. allgemeine Kontinuumshypothese besagt, daß für eine beliebige unendliche Menge $U$ niemals $|U|<|X|<|\mathcal{P}(U)|$ für irgendeine Menge $X$ gilt. (Darin ist $(\mathrm{KH})$ als Spezialfall $|U|=|\mathbb{N}|$ enthalten.) Da dies für endliche Mengen offensichtlich falsch ist, verlangt bereits die spezielle Kontinuumshypothese eine drastische Reduktion der durch Potenzmengenbildung verursachten Kardinalitätssteigerung beim Übergang vom Endlichen zum Unendlichen. Während im Finiten der Potenzmengensprung die Mächtigkeit exponentiell vergrößert $\left(\mathcal{P}(M)\right.$ hat genau $2^{n}$ Elemente, wenn $M$ genau $n$ Elemente hat), gestattet die allgemeine Kontinuumshypothese eine Erhöhung der Mächtigkeit im Infiniten sozusagen um nur eine Einheit. (So wie sich zwischen 100 und 101 keine natürliche Zahl einschieben läßt, soll man zwischen die Mächtigkeiten $|U|$ und $|\mathcal{P}(U)|$ keine andere Mächtigkeit $|X|$ einschieben können.) Vom finiten Standpunkt ist die Kontinuumshypothese eigentlich absurd. Andererseits ist ein finiter Standpunkt dem Unendlichen gegenüber nicht viel weniger als absurd.

Gerade im Zusammenhang mit der Potenzmengenbildung gibt es ein ökonomisches Argument, das für die allgemeine Kontinuumshypothese spricht. Im Finiten ist die Kardinalitätssteigerung um eine Einheit auf natürliche und einfache Weise durch Hinzunahme eines fremden Elements erzielbar: Die Menge $M \cup\{a\}$ ist im Falle $a \notin M$ mächtiger als die endliche Menge $M$. DeDEKIND sah es geradezu als Definition des Unendlichen an, daß dies bei unendlichen Mengen nicht funktioniert: Für unendliche Mengen $U$ gilt stets $|U \cup\{a\}|=|U|$. Die einfachste mengentheoretische Operation, mit der man aus einer unendlichen Menge $U$ eine Menge erzeugen kann, die mächtiger ist als die Menge $U$ selbst, ist die Bildung der Potenzmenge $\mathcal{P}(U)$. In diesem Sinn trägt die allgemeine Kontinuumshypothese der Einfachheit der Potenzmengenbildung Rechnung.

\section{Alternativen zur Kontinuumshypothese}

Nach den berühmten Resultaten von GöDEL und COHEN ist (KH) unabhängig von einem Standardaxiomensystem der Mengenlehre (vgl. [10]). Bezeichnet man mit (ZFC) das bekannteste mengentheoretische Axiomensystem, nämlich das von ZERMELO und FRAENKEL (inklusive Auswahlaxiom), so heißt das, daß (KH) innerhalb von (ZFC) weder beweisbar noch widerlegbar ist, genauer: Unter der Annahme, daß (ZFC) widerspruchsfrei 
ist, bleibt diese Widerspruchsfreiheit erhalten, wenn man (ZFC) um (KH) als Zusatzaxiom erweitert, aber die Widerspruchsfreiheit bleibt auch erhalten, wenn man (ZFC) die Negation von $(\mathrm{KH})$ als weiteres Axiom hinzufügt. Wir betrachten nun folgende Alternativen $\mathrm{zu}(\mathrm{KH})$

(KH1) Es gibt eine Menge $R_{1}$ mit $\mathbb{N} \subset R_{1} \subset \mathbb{R}$ und $|\mathbb{N}|<\left|R_{1}\right|<|\mathbb{R}|$, so daß gilt: Wenn $\mathbb{N} \subset X \subset \mathbb{R}$, dann gilt stets $|X|=|\mathbb{N}|$ oder $|X|=\left|R_{1}\right|$ oder $|X|=|\mathbb{R}|$.

Im Zusammenhang mit der Hypothese (KH1), die natürlich mit $(\mathrm{KH})$ unvereinbar ist, gibt es eine Reihe bemerkenswerter Resultate (vgl. [2], [10]). Da auch (KH1) unabhängig von (ZFC) ist, stellt (KH1) eine interessante Alternative zu (KH) dar. Allgemein kann man zeigen, daß für jede natürliche Zahl $n$ die folgende Behauptung (KHn) unabhängig von (ZFC) ist:

$(\mathrm{KH} n)$ Es gibt Mengen $R_{1}, R_{2}, \ldots, R_{n}$ mit $\mathbb{N} \subset R_{1} \subset R_{2} \subset \cdots \subset R_{n} \subset \mathbb{R}$ und $|\mathbb{N}|<\left|R_{1}\right|<\left|R_{2}\right|<\cdots<\left|R_{n}\right|<|\mathbb{R}|$, so daß gilt: Wenn $\mathbb{N} \subset X \subset \mathbb{R}$, dann gilt stets $|X|=|\mathbb{N}|$ oder $|X|=|\mathbb{R}|$ oder $|X|=\left|R_{m}\right|$ für ein $m=1,2, \ldots, n$.

Salopp gesprochen bedeutet (KHn), daß es zwischen der Mächtigkeit des Abzählbaren und des Kontinuums genau $n$ verschiedene Mächtigkeiten gibt. Insbesondere ist (KHn) für $n=0$ identisch mit (KH). Mit (KHn) für $n=0,1,2, \ldots$ liegen somit unendlich viele von (ZFC) unabhängige Hypothesen verwandten Charakters vor, so daß man verleitet sein könnte, die Gültigkeit von $(\mathrm{KH})$ als eine reine Geschmacksfrage anzusehen: Für jedes $n$ liefert die Kombination (ZFC) plus (KHn) eine eigene der Mathematik zugrunde liegende Mengenlehre und es ist kein Konflikt mit der Logik zu befürchten, wenn man eine dieser unendlich vielen Mengenlehren als das ,wahre“ Fundament der Mathematik ansehen will.

\section{Eine eigenartige Ordnung des Zahlenkontinuums}

Wie üblich nennen wir eine binäre Relation $\prec$ auf einer Menge $M$ eine (strikte) Totalordnung, wenn $\prec$ irreflexiv und transitiv ist, und für je zwei verschiedene Elemente $x, y \in M$ entweder $x \prec y$ oder $y \prec x$ gilt. Mit bekannten Instrumenten der ordinalen Mengentheorie kann man auf jeder Menge $M$ eine Totalordnung $\prec$ festlegen, so daß die Menge $M$ mächtiger ist als jeder Abschnitt $\{x \mid x \prec a\}(a \in M)$. Unter der Annahme von (KH) gilt daher die Aussage

$\left(\mathrm{KH}^{\prime}\right) \quad$ Es gibt eine Totalordnung $\prec$ auf $\mathbb{R}$, so daß $\{x \in \mathbb{R} \mid x \prec$ a für alle $a \in \mathbb{R}$ abzählbar ist.

Tatsächlich ist diese Aussage sogar äquivalent mit $(\mathrm{KH})$, da die Implikation $\left(\mathrm{KH}^{\prime}\right) \Longrightarrow$ (KH) folgendermaßen bewiesen werden kann: Es sei $\prec$ irgendeine Totalordnung auf $\mathbb{R}$. Angenommen, $(\mathrm{KHb})$ ist falsch. Dann gibt es eine überabzählbare Teilmenge $U$ von $\mathbb{R}$ mit $|U|<|\mathbb{R}|$. Wir betrachten nun die Menge

$$
T:=U \cup \bigcup_{u \in U}\{x \in \mathbb{R} \mid x \prec u\}
$$

und unterscheiden die beiden Fälle $|T|>|U|$ und $|T|=|U|$. (Trivialerweise ist $|T|<|U|$ unmöglich.) Der Fall $|T|>|U|$ kann offensichtlich nur dann eintreten, wenn irgendein 
Abschnitt $\{x \in \mathbb{R} \mid x \prec u\}$ mächtiger als $U$ ist. Im Falle $|T|=|U|$ ist $\mathbb{R} \backslash T \neq \emptyset$ wegen $|U|<|\mathbb{R}|$ gesichert. So wie die Menge $T$ gebildet wurde, muß für $a \in \mathbb{R} \backslash T$ jedenfalls $U \subset\{x \in \mathbb{R} \mid x \prec a\}$ gelten. In beiden Fällen muß also der Abschnitt $\{x \in \mathbb{R} \mid x \prec a\}$ für irgendein $a \in \mathbb{R}$ überabzählbar sein und somit $\left(\mathrm{KH}^{\prime}\right)$ falsch sein.

\section{Sierpinski-Mengen in der Euklidischen Ebene}

Wir nennen eine Teilmenge $\mathcal{D}$ des $\mathbb{R}^{2}$ eine Sierpinski-Menge (siehe auch [15]), wenn jede zur $x$-Achse parallele Gerade die Menge $\mathcal{D}$ in nur abzählbar vielen Punkten schneidet und jede zur $y$-Achse parallele Gerade die Menge $\mathcal{D}$ in nur abzählbar vielen Punkten meidet:

(1) Für alle $y \in \mathbb{R}$ ist die Menge $\{x \in \mathbb{R} \mid(x, y) \in \mathcal{D}\}$ abzählbar.

(2) Für alle $x \in \mathbb{R}$ ist die Menge $\{y \in \mathbb{R} \mid(x, y) \notin \mathcal{D}\}$ abzählbar.

Gibt es Sierpinski-Mengen? Nun, die Antwort auf diese Frage steht und fällt mit der Kontinuumshypothese! Tatsächlich ist die Existenz von Sierpinski-Mengen äquivalent mit $(\mathrm{KH})$, was wir unter Berufung auf das vorige Kapitel in zwei Schritten erledigen.

Satz 1. Wenn $\prec$ eine wie durch $\left(\mathrm{KH}^{\prime}\right)$ beschriebene Totalordnung auf $\mathbb{R}$ ist, dann ist $\left\{(x, y) \in \mathbb{R}^{2} \mid x \prec y\right\}$ eine Sierpinski-Menge.

Beweis. (1) ist für $\mathcal{D}=\left\{(x, y) \in \mathbb{R}^{2} \mid x \prec y\right\}$ per definitionem erfüllt. (2) bekommt man wegen $\mathbb{R}^{2} \backslash \mathcal{D}=\left\{(x, y) \in \mathbb{R}^{2} \mid y \prec x\right\} \cup\{(x, x) \mid x \in \mathbb{R}\}$ ebenfalls sofort.

Satz 2. Wenn (KH) falsch ist, dann kann es keine Sierpinski-Menge geben.

Beweis. Es sei $Y$ eine überabzählbare Teilmenge von $\mathbb{R}$ mit $|Y|<|\mathbb{R}|$. (Wenn (KHb) falsch ist, muß es eine solche Menge geben.) Um nun die Nichtexistenz von Sierpinski-Mengen zu bestätigen, sei $A$ irgendeine Teilmenge von $\mathbb{R}^{2}$, so daß $\{x \in \mathbb{R} \mid(x, y) \in A\}$ abzählbar für alle $y \in \mathbb{R}$ ist. Wir zeigen nun, daß dann niemals auch $\{y \in \mathbb{R} \mid(x, y) \notin A\}$ abzählbar für alle $x \in \mathbb{R}$ sein kann. Dazu betrachten wir die Menge

$$
X=\bigcup_{y \in Y}\{x \in \mathbb{R} \mid(x, y) \in A\}
$$

und stellen fest, daß $X$ als Vereinigung abzählbarer Mengen höchstens dieselbe Mächtigkeit wie $Y$ haben kann: $|X| \leq|Y|$. Wegen $|Y|<|\mathbb{R}|$ ist dann $\mathbb{R} \backslash X \neq \emptyset$, so daß wir irgendein $x \in \mathbb{R} \backslash X$ auswählen können. Für dieses $x$ gilt dann $(x, y) \notin A$ für jedes $y \in Y$. Somit ist $\{y \in \mathbb{R} \mid(x, y) \notin A\}$ eine Obermenge von $Y$ und daher überabzählbar.

\section{Der Satz von Fubini}

Mit den Sierpinski-Mengen aus dem vorigen Kapitel gelangt man zu einer interessanten Aussage über Doppelintegrale. Es sei $\mathcal{Q}=[0,1]^{2}$ das Einheitsquadrat im $\mathbb{R}^{2}$ und $f$ eine reellwertige, nichtnegative Funktion auf $\mathcal{Q}$. Im Falle, daß $f$ Lebesgue-meßbar ist, gilt bekanntlich

$$
\iint_{\mathcal{Q}} f(x, y) \mathrm{d}(x, y)=\int_{0}^{1}\left(\int_{0}^{1} f(x, y) \mathrm{d} y\right) \mathrm{d} x=\int_{0}^{1}\left(\int_{0}^{1} f(x, y) \mathrm{d} x\right) \mathrm{d} y .
$$


Ist $f$ nicht Lebesgue-meßbar, so kann zwar das Doppelintegral naturgemäß nicht gebildet werden, es könnten aber die beiden iterierten Integrale trotzdem existieren. Ein Beispiel einer solchen Funktion erhält man, wenn man nach SIERPINSKI [13] eine nichtmeßbare Teilmenge $\mathcal{E}$ der Ebene $\mathbb{R}^{2}$ konstruiert, so daß jede Gerade in $\mathbb{R}^{2}$ die Menge $\mathcal{E}$ in höchstens zwei Punkten schneidet. Die Indikatorfunktion $\chi_{\mathcal{E}}$ (also die durch $\chi_{\mathcal{E}}(x, y)=1$ für $(x, y) \in \mathcal{E}$ und $\chi_{\mathcal{E}}(x, y)=0$ für $(x, y) \notin \mathcal{E}$ definierte Funktion) ist dann auf $\mathcal{Q}$ nicht Lebesgue-meßbar, die iterierten Integrale jedoch existieren beide und natürlich gilt

$$
\int_{0}^{1}\left(\int_{0}^{1} \chi_{\mathcal{E}}(x, y) \mathrm{d} y\right) \mathrm{d} x=\int_{0}^{1}\left(\int_{0}^{1} \chi_{\mathcal{E}}(x, y) \mathrm{d} x\right) \mathrm{d} y=0 \text {. }
$$

Naheliegende Frage: Müssen die beiden iterierten Integrale einer nicht meßbaren Funktion, wenn sie existieren, identisch sein? Der folgende Satz von SIERPINS KI [14] gibt eine interessante Teilantwort.

Satz 3. Unter der Annahme von (KH) gibt es eine Funktion $f: \mathcal{Q} \rightarrow[0,1]$ dergestalt, $d a \beta \int_{0}^{1} f(x, y) \mathrm{d} y=1$ für alle $x \in[0,1]$ und $\int_{0}^{1} f(x, y) \mathrm{d} y=0$ für alle $y \in[0,1]$ gilt. Insbesondere gilt $\int_{0}^{1}\left(\int_{0}^{1} f(x, y) \mathrm{d} y\right) \mathrm{d} x=1$ und $\int_{0}^{1}\left(\int_{0}^{1} f(x, y) \mathrm{d} x\right) \mathrm{d} y=0$, so daß $f$ in extremer Weise nicht Lebesgue-meßbar ist.

Beweis. Man nehme eine Sierpinski-Menge $\mathcal{D}$, bilde $\mathcal{B}=\mathcal{D} \cap \mathcal{Q}$ und setze $f=\chi_{\mathcal{B}}$. Dann gilt für jedes feste $y \in[0,1]$ die Gleichung $f(x, y)=0$ für fast alle $x \in[0,1]$ und für jedes feste $x \in[0,1]$ die Beziehung $f(x, y)=1$ für fast alle $y \in[0,1]$.

Im Gegensatz zur Existenz einer Sierpinski-Menge ist die Existenz einer Funktion $f$ wie in Satz 3 nicht äquivalent zu (KH). Es könnte so eine Funktion geben, auch wenn (KH) falsch ist. Die Existenz einer solchen Funktion ist aber ebenfalls unabhängig von (ZFC), wie FRIEDMAN [9] gezeigt hat. Betrachten wir im Zusammenhang mit dem Satz von FUBINI die Aussage

Wenn $f: \mathbb{R}^{2} \rightarrow \mathbb{R}$ beschränkt ist, so daß die Integrale

$$
\int_{-\infty}^{\infty}\left(\int_{-\infty}^{\infty} f(x, y) \mathrm{d} y\right) \mathrm{d} x \quad \text { und } \int_{-\infty}^{\infty}\left(\int_{-\infty}^{\infty} f(x, y) \mathrm{d} x\right) \mathrm{d} y
$$

beide existieren, dann stimmen die beiden Integrale überein.

Nach Satz 3 steht (AI) in Widerspruch zu (KH). Man weiß aber, daß (AI) nicht in Widerspruch zu (KH1) aus Kapitel 3 steht. Tatsächlich folgt aus Resultaten von SoLOVAY und FREILING (vgl. [2]), daß (AI) unabhängig von dem um (KH1) erweiterten Axiomensystem (ZFC) ist. Die Aussage (AI) ist äquivalent zu folgender Aussage, die Mengentheoretikern als Freilings Axiom bekannt ist (vgl. [2]): 
(FA) Wenn eine Funktion auf $\mathbb{R}$ gegeben ist, die jeder reellen Zahl $x$ eine Lebesguesche Nullmenge $\mathcal{N}_{x} \subset \mathbb{R}$ mit $x \notin \mathcal{N}_{x}$ zuordnet, dann gibt es jedenfalls zwei reelle Zahlen a und $b$ dergestalt, daß $a \notin \mathcal{N}_{b}$ und $b \notin \mathcal{N}_{a}$ gilt.

Via (FA) sieht man auch direkt, daß (AI) mit (KH) nicht verträglich ist. Ist nämlich $\prec$ eine Totalordnung auf $\mathbb{R}$ gemäß $\left(\mathrm{KH}^{\prime}\right)$, dann ist durch $x \mapsto \mathcal{N}_{x}:=\{y \in \mathbb{R} \mid y \prec x\}$ eine Funktion von $\mathbb{R}$ in die Menge aller Lebesgueschen Nullmengen gegeben, da $\mathcal{N}_{x}$ für alle $x \in \mathbb{R}$ abzählbar ist. Es gilt dann $x \notin \mathcal{N}_{x}$ für alle $x \in \mathbb{R}$, aber es kann kein Paar verschiedener $a, b \in \mathbb{R}$ mit $a, b \notin \mathcal{N}_{a} \cup \mathcal{N}_{b}$ geben. (Für $a \neq b$ gilt offensichtlich $a \notin \mathcal{N}_{b}$ genau dann, wenn $b \in \mathcal{N}_{a}$.)

Ersetzt man in (FA) „Lebesguesche Nullmenge“ durch „nirgends dichte Menge“, so gewinnt man eine Aussage, die nicht nur mit $(\mathrm{KH})$ verträglich, sondern sogar allgemein gültig ist. (Nach BAGEMIHL [1] gibt es dann sogar eine dichte Teilmenge $D$ von $\mathbb{R}$, so daß $a, b \notin \mathcal{N}_{a} \cup \mathcal{N}_{b}$ für alle $a, b \in D$ gilt.) Dies verträgt sich auch blendend mit einer topologischen Sichtweise, nach der nirgends dichte Mengen besonders klein sind. Lebesguesche Nullmengen dagegen können topologisch betrachtet durchaus riesige Ausmaße annehmen. So gibt es bekanntlich (vgl. [11]) eine Lebesguesche Nullmenge $L \subset \mathbb{R}$ dergestalt, daß $\mathbb{R} \backslash L$ eine nur abzählbare Vereinigung nirgends dichter Teilmengen von $\mathbb{R}$ ist. Für den Topologen ist somit das Axiom (FA) eher unplausibel, während der Maßtheoretiker, dem Lebesguesche Nullmengen grundsätzlich winzig erscheinen, sich leichter mit (FA) anfreunden könnte, dann aber $(\mathrm{KH})$ als falsch ansehen müßte.

\section{Maße auf dem Kontinuum}

Es sei $I=[a, b]$ (mit $a<b$ ) irgendein kompaktes Intervall der Zahlengeraden $\mathbb{R}$, vorzugsweise $I=[0,1]$. Eine Funktion $\mu$, die jeder Teilmenge $X$ des Intervalls $I$ eine nichtnegative reelle Zahl $\mu(X)$ zuweist, wollen wir ein Maß auf I nennen, wenn $\mu(E)=0$ für alle endlichen Mengen $E \subset I$ gilt und

$$
\mu\left(\bigcup_{n=1}^{\infty} A_{n}\right)=\sum_{n=1}^{\infty} \mu\left(A_{n}\right)
$$

für alle Folgen $A_{1}, A_{2}, A_{3}, \ldots$ paarweise disjunkter Teilmengen von $I$ gilt. Insbesondere gilt stets $\mu(A \cup B)=\mu(A)+\mu(B)$ für disjunkte Mengen $A, B \subset I$ und daher auch $\mu(X) \leq \mu(Y)$ für $X \subset Y \subset I$. (Man setze $A=A_{1}, B=B_{2}$ und $A_{n}=\emptyset(n \geq 3)$, sowie $A=X$ und $B=Y \backslash X$.) Ferner stellen wir fest, daß $\mu(A)=0$ für alle abzählbaren Mengen $A \subset I$ gilt. Das folgt sofort aus $(*)$ wegen $A=\bigcup_{a \in A}\{a\}$ und $\mu(\{x\})=0$ für alle $x \in I$. Außerdem folgt aus $(*)$, daß im Falle $\mu(F)=0$ für alle Mengen $F$ einer abzählbaren Familie $\mathcal{F} \subset \mathcal{P}(I)$ auch $\mu\left(\bigcup_{F \in \mathcal{F}} F\right)=0$ gilt. Schließlich ist jede Familie $\mathcal{F}$ paarweise disjunkter Teilmengen von $I$, wo $\mu(X)>0$ für alle $X \in \mathcal{F}$ gilt, abzählbar. (Da $\mu(X) \leq \mu(I) \in \mathbb{R}_{+}$für alle $X \subset I$ gilt, $\operatorname{mu}\left\{X \in \mathcal{F} \mid \mu(X) \geq \frac{1}{n}\right\}$ wegen $(*)$ für alle ganzen $n \geq 1$ endlich sein. Wegen $\mathcal{F}=\bigcup_{n=1}^{\infty}\left\{X \in \mathcal{F} \mid \mu(X) \geq \frac{1}{n}\right\}$ muß daher $\mathcal{F}$ abzählbar sein.) 
Die Bezeichnung Maß kommt daher, daß ein Wahrscheinlichkeitsmaß $\mu$ auf dem Intervall $[0,1]$ eine Funktion mit analogen Eigenschaften (und zusätzlich $\mu([0,1])=1$ ) ist, die allerdings nur gewisse Teilmengen von $[0,1]$ als Argument zuläßt. Unser Maß hingegen soll auf allen Teilmengen von $I$ definiert sein. Ein naheliegendes, triviales Beispiel ist das Nullmaß $\mu_{0}$, das durch $\mu_{0}(X)=0$ für alle $X \subset I$ definiert ist. Es gilt nun folgender Satz von ULAM [16]:

Satz 4. Unter der Annahme der Kontinuumshypothese gibt es außer $\mu_{0}$ kein Maß auf I.

Beweis. Unter der Annahme von (KH) gibt es (vgl. Kapitel 4) eine Totalordnung $\prec$ auf $\mathbb{R}$, so daß $\{x \in \mathbb{R} \mid x \prec a\}$ für alle $a \in \mathbb{R}$ abzählbar ist. Mit einer Bijektion zwischen $\mathbb{R}$ und $I$ ( $\mathbb{R}$ und $I$ sind natürlich gleichmächtig) läßt sich diese Totalordnung auf $I$ übertragen, kurz: Es gibt auch eine Totalordnung $\prec$ auf $I$, so daß $\{z \in I \mid z \prec y\}$ für alle $y \in I$ abzählbar ist. Somit kann man für alle $y \in I$ eine Injektion $g_{y}$ von $\{z \in I \mid z \prec y\}$ nach $\mathbb{N}$ definieren. Für jedes $a \in I$ und jedes $n \in \mathbb{N}$ betrachten wir nun die durch

$$
I(a, n):=\left\{b \in I \mid a \prec b \wedge g_{b}(a)=n\right\}
$$

definierte Teilmenge von $I$. Wegen der Injektivität aller $g_{y}$ gilt offensichtlich

(1) Wenn $n \in \mathbb{N}$, dann gilt stets $I(a, n) \cap I\left(a^{\prime}, n\right)=\emptyset$ für verschiedene $a, a^{\prime} \in I$.

Ferner gilt

(2) Für alle $a \in I$ ist die Differenzmenge $I \backslash \bigcup_{n \in \mathbb{N}} I(a, n)$ abzählbar.

Ist nämlich $y \notin \bigcup_{n \in \mathbb{N}} I(a, n)$, dann ist $a \prec y$ unmöglich, da sonst $y \in I\left(a, g_{y}(a)\right)$ gelten würde. Daher liegt $y$ in der abzählbaren Menge $\{x \in I \mid x \prec a\} \cup\{a\}$.

Es sei nun $\mu$ ein Maß auf $I$. Betrachtet man dazu die Menge $\{a \in I \mid \mu(I(a, n))>0\}$ für festes $n \in \mathbb{N}$, so muß sie wegen (1) abzählbar sein. Daher ist auch die Menge $\{(a, n) \in$ $I \times \mathbb{N} \mid \mu(I(a, n))>0\}$ abzählbar. Diese Menge muß aber, da $I$ überabzählbar ist, Teilmenge von $\{(a, n) \in I \times \mathbb{N} \mid a \prec b\}$ mit irgendeinem $b \in I$ sein. (Man wähle $b$ aus der Menge $I \backslash\{x \in I \mid \exists(a, n) \in I \times \mathbb{N}: \mu(I(a, n))>0 \wedge x \prec a\}$ !) Für so ein $b$ gilt dann aber $\mu(I(b, n))=0$ für alle $n \in \mathbb{N}$ und daher auch $\mu\left(\bigcup_{n \in \mathbb{N}} I(b, n)\right)=0$. Da das Maß $\mu$ auf abzählbaren Mengen verschwindet, muß wegen (2) somit $\mu(I)=0$ gelten. Damit ist $\mu$ als das Nullmaß entlarvt, da $\mu(I)=0$ automatisch $\mu(X)=0$ für alle $X \subset I$ nach sich zieht.

$\mathrm{Da} \beta \mu_{0}$ das einzige Maß auf $I$ ist, folgt erstaunlicherweise nicht nur aus $(\mathrm{KH})$, sondern auch aus jeder der Aussagen (KHn) (mit $n \in \mathbb{N}$ beliebig) aus Kapitel 3. Wie Ulam zeigen konnte, ist auch dann $\mu_{0}$ das einzige Maß auf $I$, wenn man lediglich annimmt:

(U) Wenn $X$ eine überabzählbare Teilmenge von $\mathbb{R}$ ist, dann läßt sich $X$ nicht als Vereinigung $\bigcup_{\lambda \in \Lambda} X_{\lambda}$ mit Mengen $X_{\lambda} \subset X$ schreiben, so daß sowohl $\left|X_{\lambda}\right|<|X|$ für alle $\lambda \in \Lambda$ als auch $|\Lambda|<|X|$ für die Indexmenge $\Lambda$ selbst gilt.

Natürlich folgt (U) sofort aus (KH). Offensichtlich wird (U) aber auch von jeder der Aussagen $(\mathrm{KH} n)($ mit $n \in \mathbb{N}$ beliebig) impliziert. 


\section{Summen- und Differenzendarstellungen reeller Zahlen}

Eine Äquivalenzrelation $\sim$ auf $\mathbb{R}$ wollen wir summenfinit nennen, wenn es für jede reelle Zahl $x$ nur endlich viele Darstellungen $x=a+b$ mit äquivalenten Zahlen $a$ und $b$ gibt: Für alle $x \in \mathbb{R}$ ist die Menge $\left\{(a, b) \in \mathbb{R}^{2} \mid a \sim b \wedge x=a+b\right\}$ endlich. Wie üblich sei die Quotientenmenge $\mathbb{R} / \sim$ die Menge $\{\{u \in \mathbb{R} \mid u \sim a\} \mid a \in \mathbb{R}\}$ aller Äquivalenzklassen. CIESIELSKI und LARSON [5] konnten eine summenfinite Äquivalenzrelation $\sim$ auf $\mathbb{R}$ konstruieren, deren Quotientenmenge $\mathbb{R} / \sim$ abzählbar ist! Die Frage nach einer analogen differenzenfiniten Äquivalenzrelation hängt merkwürdigerweise engstens mit $(\mathrm{KH})$ zusammen. Zunächst kann man natürlich nicht verlangen, daß 0 nur mit endlich vielen Paaren $(a, b)$ als Differenz $a-b$ dargestellt wird. Wir nennen daher eine $\ddot{A}$ quivalenzrelation $\sim$ auf $\mathbb{R}$ differenzenfinit, wenn für jede reelle Zahl $x \neq 0$ die Menge $\left\{(a, b) \in \mathbb{R}^{2} \mid a \sim b \wedge x=a-b\right\}$ endlich ist. Es gilt nun der folgende

Satz 5. Die Kontinuumshypothese ist äquivalent zur Existenz einer differenzenfiniten Äquivalenzrelation auf $\mathbb{R}$ mit abzählbarer Quotientenmenge.

Dieser Satz von CIESIELSKI [4] steht in engem Zusammenhang mit folgendem Satz von ERDÖS und KAKUTANI [7].

Satz 6. Die Kontinuumshypothese ist äquivalent zur Existenz einer Äquivalenzrelation auf $\mathbb{R} \backslash\{0\}$ mit abzählbarer Quotientenmenge, so daß jede Äquivalenzklasse eine Basis des Vektorraumes $\mathbb{R}$ über dem Körper $\mathbb{Q}$ ist.

Für beide Sätze findet man einen (anspruchsvollen) Beweis in der hervorragenden Monographie [3] (Theorem 7.3.9 und Theorem 7.4.6).

\section{Interpolation mit ganzen Funktionen}

Es sei $\mathcal{F}$ eine Familie ganzer, also auf ganz $\mathbb{C}$ definierter analytischer Funktionen. Bekanntlich ist eine Familie komplexwertiger stetiger Funktionen auf $\mathbb{C}$ höchstens von der Mächtigkeit des Kontinuums. Insbesondere gilt für unsere Familie stets $|\mathcal{F}| \leq|\mathbb{R}|$. Wir wollen $\mathcal{F}$ nun $\omega$-wertig nennen, wenn immer nur abzählbar viele komplexe Zahlen als Werte der Funktionen aus $\mathcal{F}$ bei irgendeinem komplexen Argument auftreten können, kurz wenn die Menge $\{f(z) \mid f \in \mathcal{F}\}$ für alle $z \in \mathbb{C}$ abzählbar ist. Da ganz bekanntlich eine äußerst starke Eigenschaft einer Funktion ist, stellt sich die Frage: Können $\omega$-wertige Familien ganzer Funktionen überabzählbar sein? Die Antwort auf diese Frage ist ein wenig überraschend, da sie auf den ersten Blick in scheinbar verkehrter Weise mit der Kontinuumshypothese zusammenhängt.

Satz 7. Wenn (KH) falsch ist, dann ist jede $\omega$-wertige Familie ganzer Funktionen abzählbar. Dagegen gibt es unter Annahme von $(\mathrm{KH})$ eine $\omega$-wertige Familie $\mathcal{F}$ ganzer Funktionen von der Mächtigkeit des Kontinuums.

Dieser fabelhafte Satz stammt von ERDös, der ihn unter Zuhilfenahme der Ordinalzahlen bewiesen hat (vgl. [6]). Da die zweite Aussage des Satzes ohne Wohlordnungsargumente nicht bewiesen werden kann, begnügen wir uns abschließend damit, den von ERDös gegebenen Beweis der ersten Aussage zu vereinfachen. Zunächst zeigen wir folgendes 
Lemma. Wenn $\mathcal{F}$ eine Familie ganzer Funktionen ist, für die $|\mathcal{F}|<|\mathbb{C}|$ gilt, dann gibt es eine komplexe Zahl $z$, so daß stets $f(z) \neq g(z)$ für verschiedene $f, g \in \mathcal{F}$ gilt.

Beweis. Es sei $\mathcal{F}$ eine Familie ganzer Funktionen mit $|\mathcal{F}|<|\mathbb{C}|$. Es gilt dann natürlich auch $|\mathcal{F} \times \mathcal{F}|<|\mathbb{C}|$. Für jedes Paar $(f, g)$ verschiedener Funktionen $f, g \in \mathcal{F}$ betrachten wir die Menge $\mathcal{C}(f, g):=\{z \in \mathbb{C} \mid f(z)=g(z)\}$. Diese Menge muß immer abzählbar sein! Denn verschiedene auf einer Kreisscheibe $|z| \leq r$ analytische Funktionen können nur in endlich vielen Punkten übereinstimmen, da unendlich viele Punkte sich innerhalb der Kreisscheibe häufen müssen. Vereinigt man alle Kreisscheiben $|z| \leq r$ für $r=1,2,3, \ldots$, so bekommt man eine Übereinstimmung verschiedener ganzer Funktionen in höchstens abzählbar vielen Punkten.

Wir vereinigen nun all diese abzählbaren Mengen $\mathcal{C}(f, g)$ für $(f, g) \in \mathcal{F} \times \mathcal{F}$ mit $f \neq g$ und bekommen eine Teilmenge $\mathcal{U}$ von $\mathbb{C}$, für die klarerweise $|\mathcal{U}| \leq|\mathbb{N}|(\mathcal{F}$ darf endlich sein und auch $\mathcal{U}$ kann endlich sein) oder $|\mathcal{U}| \leq|\mathcal{F} \times \mathcal{F}|$ und somit jedenfalls $|\mathcal{U}|<|\mathbb{C}|$ gilt. So wie $\mathcal{U}$ konstruiert wurde, muß stets $f(z) \neq g(z)$ für verschiedene $f, g \in \mathcal{F}$ gelten, wenn das Argument $z$ der Menge $\mathbb{C} \backslash \mathcal{U} \neq \emptyset$ entnommen wird.

Mit Hilfe des Lemmas ist ein Beweis der ersten Aussage von Satz 7 nun schnell erbracht. Angenommen also, $(\mathrm{KH})$ ist falsch. Da $\mathbb{R}$ und $\mathbb{C}$ gleichmächtig sind, gibt es dann eine überabzählbare Teilmenge $\mathcal{D}$ von $\mathbb{C}$ mit $|\mathcal{D}|<|\mathbb{C}|$. Ist nun $\mathcal{F}$ eine $\omega$-wertige Familie ganzer Funktionen, dann kann $\mathcal{F}$ nicht überabzählbar sein. Denn sonst ließe sich aus $\mathcal{F}$ eine überabzählbare Teilfamilie $\mathcal{F}^{\prime}$ herausgreifen, für die $\left|\mathcal{F}^{\prime}\right| \leq|\mathcal{D}|$ gilt. (Im Falle $|\mathcal{F}| \leq$ $|\mathcal{D}|$ kann man natürlich $\mathcal{F}=\mathcal{F}^{\prime}$ wählen.) Wegen $|\mathcal{D}|<|\mathbb{C}|$ kann dann aber das Lemma auf die Familie $\mathcal{F}^{\prime}$ angewandt werden, so daß $\mathcal{F}^{\prime}$ nicht $\omega$-wertig sein kann. Insbesondere kann $\mathcal{F}$ selbst nicht $\omega$-wertig gewesen sein!

\section{Literatur}

[1] Bagemihl, F.: The existence of an everywhere dense independent set. Michigan Math. J. 20 (1973), 1-2.

[2] Brunner, N.: Mathematische Intuition, Kontinuumshypothese und Auswahlaxiom. Jahrbuch 1988 der Gödel-Gesellschaft, 96-101.

[3] Ciesielski, K.: Set theory for the working mathematician. Cambridge 1997.

[4] Ciesielski, K.: Sum and difference free partitions of vector spaces. Colloq. Math. 71 (1996), 263-271.

[5] Ciesielski, K.; Larson, L.: Uniformly antisymmetric functions. Real Anal. Exchange 19 (1994), $226-235$.

[6] Erdös, P.: An interpolation problem associated with the continuum hypothesis. Michigan Math. J. 11 (1964), 9-10.

[7] Erdös, P.; Kakutani, S.: On non-denumerable graphs. Bull. Amer. Math. Soc. 49 (1943), 457-461.

[8] Freiling, C.: Axioms of symmetry: Throwing darts at the real number line. J. Symbolic Logic 51 (1986), 190-200.

[9] Friedman, H.: A consistent Fubini-Tonelli theorem for non-measurable functions. Illinois J. Math. 24 (1980), 390-395.

[10] Jech, Th.: Set Theory. 3rd ed., Berlin-Heidelberg-New York 2002.

[11] Oxtoby, J.C.: Maß und Kategorie. Berlin-Heidelberg-New York 1971.

[12] Sierpinski, W.: Sur un théorème équivalent à l'hypothèse du continu. Bull. Int. Acad. Sci. Cracovie A (1919), 1-3. 
[13] Sierpinski, W.: Sur un problème concernant les ensembles mesurables superficiellement. Fund. Math. 1 (1920), 112-115.

[14] Sierpinski, W.: Sur les rapports entre l'existence des intégrales $\int_{0}^{1} f(x, y) d x, \int_{0}^{1} f(x, y) d y$ et $\int_{0}^{1} d x \int_{0}^{1} f(x, y) d y$. Fund. Math. 1 (1920), 142-147.

[15] Simms, J.C.: Sierpinski’s Theorem. Bull. Belg. Math. Soc. Simon Stevin 65 (1991), 69-163.

[16] Ulam, S.M.: Zur Maßtheorie in der allgemeinen Mengenlehre. Fund. Math. 16 (1930), 141-150.

Gerald Kuba

Institut für Mathematik

Universität für Bodenkultur

A-1180 Wien, Österreich 